
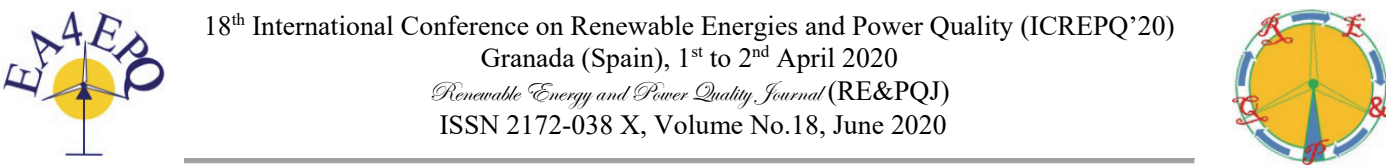

\title{
Estimating Distributed Generation reliability level
}

\author{
Vladislav O. Samoylenko ${ }^{1}$, Pavel V. Ilyushin ${ }^{2}$ and Andrew V. Pazderin ${ }^{1}$ \\ ${ }^{1}$ Department of Automated Electrical Systems \\ Ural Federal University \\ Mira str., 19, 620002 Yekaterinburg (Russia) \\ Phone/Fax number: +007 343 359-16-15, e-mail: vvsamoylenko@yandex.ru, a.v.pazderin@urfu.ru \\ 2 Petersburg Power Engineering Institute of Professional Development \\ Aviatsionnaya str., 23, 196135 Saint Petersburg (Russia) \\ Phone/Fax number: +007 812 373-61-74, e-mail: ilyushin.pv@mail.ru
}

\begin{abstract}
The paper considers the reliability level of different distributed generation units. The world comparative statistics of reliability parameters depending on the unit capacity and other influencing factors are presented. The study includes an analysis of possible approaches to the correction of statistical parameters under the particular conditions. The calculation and analysis of structural reliability parameters for a group of distributed generation units is performed. It is shown that conventional $N+1$ reliability principle is not enough for distributed generation given a great number of operation conditions. A set of $N+n$ principles is proposed according to the required distributed generation reliability level. Considering the reliability parameters is of great importance for the selection of installed power and the structure of distributed generation for industrial and household facilities under the design and evaluation of its technical and economic efficiency. Gradual growth of the share of distributed generation in power and energy balances of power systems raises the issue of its reliability calculation and regulation. The results obtained in the paper are also important for the maintenance, planning of repairs, and providing necessary logistics solution for the delivery of spare parts and consumables to generating plants.
\end{abstract}

Key words. Distributed power generation; power system reliability; power distribution; availability; statistical distributions.

\section{Introduction}

The ongoing development of Distributed Generation (DG) is concerned primarily with the renewables like photovoltaic power plants (PVPPs) and wind power plants (WPP). Distributed generation in the form of combined heat and power (CHP) is used to supply urban buildings and structures, residential households by electricity and thermal energy. In suburban and rural areas both types of sources supply energy for modern industrial enterprises and farmers. The CHP is usually represented by gas piston units (GPUs) or gas turbine units (GTUs), including microturbines.

The issue of power supply reliability, considering DG units as a main source of the energy, is of a great interest.
Such conditions correspond to limited grid capacity, microgrids' island modes, or off-grid operation conditions. Obtaining parameters such as the availability factor and the probability of a plant operation makes possible to estimate the balance features during the year. The frequency of a unit shutdown leading to the loss of $100 \%$ of its power, affects the assessment of the possibility of power balance.

Although calculations of reliability parameters in the energy sector have been conducted for a long time [1-4], there are relatively few scientific papers devoted particularly to the assessment of the structural and balance reliability of electricity and energy supply by the DG units [5-10]. The publications are aimed primarily at improving the computation techniques and not the reliability factor application itself. A few numerical statistics are mentioned in the existing publications. In addition, there's a lack of its comparative analysis.

The gradual growth of DG share in power and energy balances of power systems raises the issue of their reliability calculation and regulation. The main problem of the DG-containing balances is that the power and energy output is considered mostly from the point of view of the primary energy source forecasting (like solar irradiance or wind speed) and its availability. Reliability consideration can be of the crucial importance when turning to the distributed power supply in terms of Distributed Generation and Smart Grid concepts.

The main goal of the paper is to show that the reliability factor cannot be neglected in terms of DG contribution to power and energy balances. The paper is devoted to the complex comparative analysis of reliability parameters of various DG types depending upon its capacity and different operating conditions. The consideration reveals that DG reliability level doesn't corresponds the reliability level of the conventional power plants. Potentially it may results in a correction of power and energy reserves and service intensity. 
The results obtained in the paper are also important for the maintenance, planning of repairs and providing necessary logistics solution for the delivery of spare parts and consumables to generating plants. Taking into account the reliability parameters is critically important for the selection of installed power and the structure of distributed generation for industrial and household facilities during the design and evaluation of its technical and economic efficiency.

\section{World Statistics of DG Units Reliability}

The reliability parameters of power supply via DG can be affected both by the parameters of the generation itself and by the reliability of adjacent grid equipment. However, the first ones are the priorities for consideration. DG objects are usually installed in the point of consumption. The length of the distribution power lines is minimal and the lines themselves rarely suffer from faults. Therefore, only the equipment of the distribution switchgear and DG units affects reliability. Reliability parameters of grid equipment are several orders of value higher than for generation units [11]. In modern practice, the calculations assume the absolute reliability of $\mathrm{MV}$ small transformers, busbars, and vacuum circuit breakers. A backup energy source in the form of a grid connection may not be available, especially for thermal energy (including the buildings with photovoltaic systems where electric heating and air conditioning is used). In addition, the available grid capacity can be less than the total load power. Thus, if the DG units are disconnected, the operation of a customer load is disrupted. So, DG units reliability is the top priority for the consideration.

The collected statistics of reliability parameters of DG units are based on reviews and meta-reviews of their reliability [12-19]. The data in the sources are represented in form of duration $t_{i}$ of operation, maintenance, idle, reserve and outage. The standard data processing methods are used [4]. The collected data are reduced to the following two parameters.

1. To assess the reliability impact of the DG on the energy balance, the availability factor $A F$ is used. It shows a share of time within a unit is ready to generate electricity:

$$
A F=\frac{t-t_{S O H}-t_{F O H}}{t} \cdot 100 \%=\frac{t_{o p}+t_{r e s}}{t} \cdot 100 \%
$$

where $t$ is the considered period of time, $t_{\mathrm{SOH}}$ is the duration of scheduled outage, $t_{F O H}$ is the duration of forced outage. The numerator of a fraction can also be summed up from the time of operation $t_{o p}$ and the idle time as the reserve or backup $t_{\text {res }}$. The last value for DG units designed for the continuous generation of electricity, can be taken as zero. Then, in the context of the year:

$$
A F=\frac{t_{o p}}{8760} \cdot 100 \%
$$

2. To assess the effect of the reliability of the DG on the power balance, the mean time between forced outages $M T B F O$ is used. This is the time between the forced outages of a unit that occurs for any internal (failure, unscheduled repair, trip by technological protection) or external (trip by relay protection) reasons:

$$
M T B F O=\frac{t_{o p}}{N}
$$

where $N$ is the quantity of outages. Forced disconnection leads to the loss of $100 \%$ of the DG unit power. At the best case, it leads to the necessity for automatic resynchronization after a few dozens of seconds, at the worst - to the necessity of service by operational personnel. $M T B F O$ is also close to the $M T B I$ parameter Mean time between incidents, used in reports by some utilities. $M T B F O$ can be obtained by dispatch reports as well as by reports of automatic control system of nondispatchable DG.

The results of the statistics collection are shown in Fig. 1. To compare the reliability level of the DG and the reliability level of large-scale power plants, the parameters of equipment corresponding to modern combined-cycle plants are given. Based on the results of the analysis, the following conclusions can be made.

1. All the DG types, except the PVPPs, have an evident dependence of reliability parameters on power. For PVPPs, the decisive role is played by the degree of technology development, i.e. PV modules technical generation as well as the development of their protection and interconnection schemes, and the availability of standby (reserve) modules.

2. Large steam-powered and gas turbine equipment have the number of forced outages 5-8 times lower and $A F$ at 2$7 \%$ higher. This is caused either by the design of such equipment or by the electrical distance from the load and disturbance points in the grid. The equipment of power stations at high voltage classes is protected by relatively advanced Relay Protection and Automation (RPA) devices Also the higher qualification of the operating personnel, and smoother load schedule contributes to the result. Relatively frequent, but short-term outages of DG units caused by disturbances in the external grid and internal power supply system, RPA imperfections, etc.

3. For hydrocarbon-based DG plants, the increase in unit capacity leads to a positive effect of increasing the mean time between forced outages. Within the range of effective capacities, this value is about $750 \mathrm{~h}$ per MW for gas piston units and $100 \mathrm{~h}$ per MW for gas turbines proportionally to their power. This is caused both by a more sophisticated design of powerful units and by more stable operating conditions.

4. The $A F$ is stable at the level of $\sim 97.5 \pm 1 \%$ for gas piston units, and for gas turbines it decreases by $0.2 \%$ per MW, due to the inaccessibility of such units repair wherever excepting the manufacturer plant. 


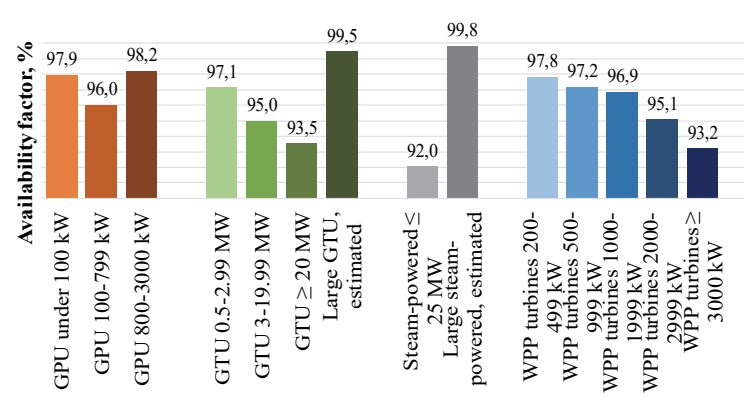

a)
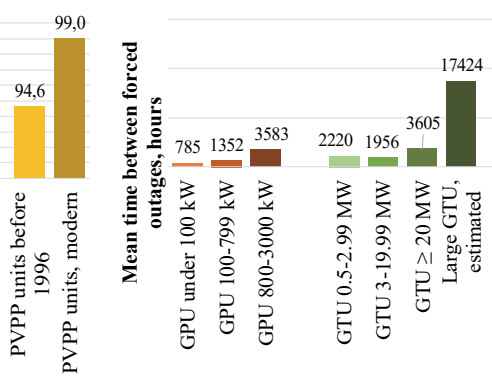

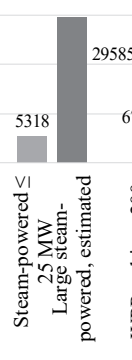

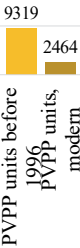

b)

Fig. 1. The bar charts of DG units reliability parameters: a) availability factor $A F$; b) mean time between forced outages $M T B F O$

5. Due to the complexity of the design and the remote location of modern wind turbines from consumption centers a step up of unit capacity leads to the reduction in $M T B F O$ and $A F$ of $1000 \mathrm{~h}$ per MW and $0.4 \%$ per MW, correspondingly.

6. The mean time between forced outages for PVPP units falls by a factor of 5 in a retrospective mainly due to faults and excessive protection trips of modern power converters. However, the development of the technologies for the PVPPs and their mass production provided the availability of such stations up to $99 \%$, which corresponds to the level of large power plants.

It should be noted, in terms of the probabilistic approach, the $A F$ can be interpreted with some assumption as the probability of DG unit operation $A F \approx p[\%]$ or $A F \approx P$ [\%], that is, $A F$ is often considered as a general statistical parameter, while $p$ and $P$ associated with the calculated value of the particular unit or power plant, correspondingly. To calculate the combinations of operation and outage probabilities for several units during a year using the described above statistics, a binomial distribution is used:

$$
q(n, k)=C_{n}^{k} q^{k} p^{n-k},
$$

where $q(n, k)$ is the probability of a state when $k$ of $n$ units are disconnected; $C_{n}{ }^{k}$ is the number of combinations of $n$ elements over $k ; q$ and $p$ is the probability of outage time and operation of a single unit, correspondingly. The value $q$ can be easily found using $q=1-p$. The cumulative probability defines the probability that the number of units under maintenance will not exceed the quantity necessary for a load operation, taking into account the available power:

$$
Q(n, k)=\sum_{j=1}^{k} q(n, j)
$$

The corresponding values of the total power can be obtained by multiplying the unit power by the number of operating or disconnected units. The issues of the statistics applicability for specific operation conditions are discussed below.

\section{Correction of the Collected Statistics}

An important factor affecting the actual reliability parameters is a technical generation of equipment.
Technical generation assumes homogeneous equipment at a certain stage of its development, significantly differing in technical and economic indicators, reliability, functionality, and others from the previously produced equipment. New generation of power plants may differ in a level of reliability, both positively and negatively. In the case of maintaining the level of technical complexity, the unit of new generations have larger service intervals and improved reliability parameters.

The states like Repair and Failure for distributed generation units are often difficult to separate. In their design, there are the parts and devices that require routine maintenance and replacement. In difficult conditions, they become weak links. It leads to an intermediate state such as Repair Due to Accelerated Wear and the need for an unscheduled stop of the generating unit. Since it is difficult to distinguish Failure and Repair states, a change in the service interval indirectly indicates a corresponding change in the reliability level of the unit. For example, if the manufacturer increases the service interval, this will lead to the corresponding increase in $M T B F O$ in the event that the causes of forced outages are dominated by failures associated with malfunction of such weak links. Therefore, a simple linear correction of $A F^{\text {corr }}$ can be performed using statistics on the causes of failures:

$$
\begin{aligned}
& A F^{c o r r}=\frac{t-t_{S O H}^{c o r r}-t_{F O H}^{c o r r}}{t} \cdot 100 \%= \\
& =\frac{t-t_{S O H}^{c o r r}-\left(\alpha \cdot \frac{t_{S O H}^{c o r r}}{t_{S O H}}+\beta\right)}{t} \cdot 100 \%,
\end{aligned}
$$

where $t_{S O H}^{\text {corr }}$ is the value of the service interval, different from the base one, $\alpha$ is the share coefficient of internal causes of forced outages, $\beta$ is the share coefficient of external causes of forced outages. When changing the service interval, $M T B F O$ is ambiguously changed:

$$
M T B F O^{\text {corr }} \approx M T B F O \cdot\left(\alpha \cdot \frac{t_{\text {SOH }}^{\text {corr }}}{t_{\text {SOH }}}+\beta\right)
$$

In case if the causes of failure are internal causes associated with accelerated wear, then the increase in $M T B F O$ can be approximated in proportion to the growth of the service interval with the corresponding weighting factor (0.5). The component of MTBFO determined by 
other (external) causes with a weighting factor of 1-0.5 = 0.5 , does not change. For example, with an average time between forced outages of $2000 \mathrm{~h}$ for units with a planned service interval of $2000 \mathrm{~h}$ and a percentage of accelerated wear of $50 \%$ in the causes of forced outages, an increase in the service interval for new generation units of up to $4000 \mathrm{~h}$ will lead to an increase in the expected $M T B F O$ up to $2000 *(0.5 * 4000 / 2000+0.5 * 1)=3000 \mathrm{~h}$. For the units discussed in Section 1, the service interval was 2000-4000 h, while the current DG plants operate at 4000$8000 \mathrm{~h}$ intervals [11].

The next influencing factor is the electrical state conditions. They are taken into account in the paper by the fractional coefficient $\beta$ of forced outages for external reasons. Frequent trips of the GPU from RPA devices with external short-circuits occur due to the low ridethrough settings for the over/under voltage and frequency devices. The corresponding average $U$ and $f$ are typically $\pm 10 \%$ and $\pm 1 \mathrm{~Hz}$, respectively. The first stage of protection trips a generator with a time delay of 0-200 ms, the second stage trips with a delay of $0.5-1 \mathrm{~s}$, although Grid Code requirements often are much tolerant. The mentioned settings lead to excessive protection trips and frequent shutdown of a DG unit. The mean time between forced outages is inversely proportional to the occurrence of the states beyond the limits of the settings. The duration of the $t_{F O H}$ determining the $A F$ can be calculated based on the probable duration of such states. Calculation of the relevant parameters to be made in accordance with the theory of reliability [4].

However, the main factor leading to a deviation from global statistics is specific local operating conditions. DG operates both in the zone of centralized power supply and in islanded microgrids located in the remote areas in a wide variety of climatic conditions and electric states. This results in more frequent repairs and decreased availability of spare parts and qualified service for DG units. Relative classification of operating conditions (OCs) of DG units is given in Table I. To match the particular operating conditions with the defined in Table I a simple method of expert evaluations was used. Easy or hard OCs score if the real parameters match the corresponding group of OCs. If two groups correspond to the easy OCs and two correspond to the hard OCs, the OCs classified as medium. Easy OCs correspond to the number of scheduled outage hours $t_{\mathrm{SOH}}$ in the absence of forced outages. Hard OCs correspond to the maximum duration of forced outage hours $t_{F O H}$. Some improvement in the statistics is that the maintenance can be combined with the repair without adding additional scheduled outage hours.

Statistics of CHP DG units at the heavy industry taking into account the defined OCs is presented in Table II. Corresponding capacity ranges of the samples vary from $1000 \mathrm{~kW}$ to $2000 \mathrm{~kW}$ for GPUs and from the $4000 \mathrm{~kW}$ to $9000 \mathrm{~kW}$ for GTUs. For comparison, typical parameters are given for the MV overhead line of $10 \mathrm{kV}$ and of $5 \mathrm{~km}$ length. It is typical for the power supply of consumers in rural areas.
Table I. - The Criteria for DG Operation Conditions Ranking

\begin{tabular}{|c|c|}
\hline Easy OCs close to the ideal & Hard OCs \\
\hline \multicolumn{2}{|c|}{ General } \\
\hline $\begin{array}{l}\text { Correct design solutions for } \\
\text { main and auxiliary equipment }\end{array}$ & $\begin{array}{l}\text { Fit the operation plan for } \\
\text { equipment already purchased }\end{array}$ \\
\hline \multicolumn{2}{|c|}{ Electric } \\
\hline Baseload & Peaking \\
\hline No frequent starts / stops & $\begin{array}{l}\text { Daily starts / stops, incl. cold } \\
\text { ones }\end{array}$ \\
\hline $\begin{array}{l}\text { Absence of disturbances in the } \\
\text { external grid }\end{array}$ & $\begin{array}{l}\text { Frequent faults, phase breaks, } \\
\text { voltage dips }\end{array}$ \\
\hline Only grid-connected operation & Periodic operation as an island \\
\hline Smooth load curve & Sufficient load steps \\
\hline $\begin{array}{l}\text { Symmetric values of currents } \\
\text { and voltages in phases }\end{array}$ & $\begin{array}{l}\text { Unbalanced values of currents } \\
\text { and voltages in phases }\end{array}$ \\
\hline \multicolumn{2}{|c|}{ Operation } \\
\hline $\begin{array}{l}\text { Fuel of a nominal calorific } \\
\text { value without mechanical and } \\
\text { chemical impurities }\end{array}$ & $\begin{array}{l}\text { Deviation of a calorific value } \\
\text { of fuel from the calculated, } \\
\text { fuel preparation issues }\end{array}$ \\
\hline $\begin{array}{l}\text { Quality consumables and } \\
\text { lubricants }\end{array}$ & $\begin{array}{l}\text { Costs priority for consumable } \\
\text { and lubricating materials }\end{array}$ \\
\hline \multicolumn{2}{|c|}{ Service and maintenance } \\
\hline Manufactirer's personnel & Own personnel \\
\hline $\begin{array}{l}\text { Closeness of warehouses of } \\
\text { spare parts and advanced } \\
\text { logistics }\end{array}$ & $\begin{array}{l}\text { Supply of spare parts and } \\
\text { consumables on demand and } \\
\text { on request }\end{array}$ \\
\hline $\begin{array}{l}\text { The service contract and the } \\
\text { manufacturer's telemetry on } \\
\text { the units }\end{array}$ & $\begin{array}{l}\text { The on-site diagnostics on } \\
\text { demand }\end{array}$ \\
\hline $\begin{array}{l}\text { The first } 3 \text { years of operation } \\
\text { of the unit (before repairs) }\end{array}$ & $\begin{array}{l}\text { Statistics for the period of } \\
\text { operation taking into account } \\
\text { repairs (from } 3 \text { years) }\end{array}$ \\
\hline $\begin{array}{l}\text { Experience in the maintenance } \\
\text { of power plants }\end{array}$ & $\begin{array}{l}\text { Experience in maintenance } \\
\text { primarily for a grid equipment } \\
\text { or an electric motors }\end{array}$ \\
\hline Proper maintenance standards & - \\
\hline
\end{tabular}

Mean time between forced outages varies from 1338 hours for GPUs to 2517 hours for GTUs. The average $M T B F O$ is 2030 hours. Calculated on the basis of the statistics share ratio of internal causes for forced outages $\alpha$ is $32.5 \%$, the share ratio $\beta$ of external causes during forced outages is $67.5 \%$. The following features are noted.

Table II. - The Statistics of DG Outages

\begin{tabular}{|c|r|r|r|r|}
\hline \multirow{2}{*}{ Equipment } & \multicolumn{4}{|c|}{ Probability } \\
\cline { 2 - 5 } & \multicolumn{2}{|c|}{$\%$} & \multicolumn{2}{|c|}{ Hours per year } \\
\cline { 2 - 5 } & Operates & \multicolumn{1}{c|}{ Down } & Operates & \multicolumn{1}{c|}{ Down } \\
\hline 10 kV overhead & 99.18 & 0.82 & 8688 & 72 \\
\hline GTU, hard OCs & 91.58 & 8.42 & 8022 & 738 \\
GTU, med OCs & 94.95 & 5.05 & 8318 & 443 \\
GTU, easy OCs & 98.32 & 1.68 & 8613 & 147 \\
\hline GPU, hard OCs & 56.93 & 43.07 & 4987 & 3773 \\
GPU, med OCs & 91.32 & 8.68 & 8000 & 760 \\
GPU, easy OCs & 97.72 & 2.28 & 8560 & 200 \\
\hline
\end{tabular}

1. Equipment availability factors in case of the most hard OCs are reduced by $30 \%$. The reason is primarily the service availability and quality of service equipment not always providing the quick elimination of the malfunction. In addition, the statistics do not contain data on gas turbine units meeting the scheduled 60-hours maintenance time $(A F=99.3 \%)$ declared by many manufacturers. 
2. The average $M T B F O$ generally corresponds to the global one. Given the high proportion of forced outages due to external causes, $\beta=67.5 \%$, it can be assumed that the main reason for the forced outages of DG units worldwide is the excessive trips of generator by protections aimed at equipment security.

In addition, it can be concluded that the reliability of the power supply by the DG units can be significantly worsened with unsatisfactory operation statistics. The corresponding calculations are presented below.

\section{Case Study}

The case study based on the statistics presented above. Different types of DG units are considered: GTUs, GPUs and WPPs. The case of PVPP including 2500 modules with a total area of $2500 \mathrm{~m}^{2}$ is calculated. The numerical series calculations were carried out using (5), (6).

For GTUs and GPUs the corrected statistics data is used (Table II) and the rated power: $6000 \mathrm{~kW}$ for GTU and 2000 for GPU, correspondingly. The reliability parameters of $q$ are taken from the third column of Table II (see above). For the renewables the world statistics from Table I was taken. The $q$ value was obtained as $q=1-p$ with the respect of the discussed above assumption of $A F \approx p[\%]$. The calculations use the most urgent wind turbine capacity of $2000-2999 \mathrm{~kW}, q=4.9 \%$. In addition, the performance of a PVPP with an installed capacity of 500 $\mathrm{kW}$ is estimated, $q=1.0 \%$. For hydrocarbon-based and WPP the results for the most typical case of power plant including four units are presented. The results of the calculations are shown in Fig. 2. For comparison, the results of rural $10 \mathrm{kV}$ overhead line [20] are presented. According to the results of the calculations, the following conclusions can be made.

The standard for electric power industry principle $N+1$, is not truly correct to the DG units. The principle assumes that for any power system equipment unit there is another one unit providing backup or reserve enough to ensure generation, transmission or distribution. Historically, it has been applied to grid equipment (transmission lines, transformers, busbars), that have a failure rate of 103-106 times less than the failure rate of generator units (i.e. the probability of events such as failure of the first unit plus failure of the second unit is minimal).

Generators strictly require periodic maintenance instead the repair on demand (the probability of events such as scheduled outage of the first plus failure of the second raises). Taking into account the real reliability parameters of the grid equipment, the $N+1$ principle allows to ensure the absence of a power deficit with a probability of at least $95.4 \%$, more often $99.7 \%$ (engineering criteria for two and three standard deviations $\sigma$ for normal distribution, respectively). The probability of the $10 \mathrm{kV}$ overhead line operation is $96.76 \%$, and the probability that the number of switched off power lines does not exceed single line is $99.96 \%$.

The application of the $N+1$ principle to generation units leads to incomplete accounting of the probability of outage time of two or more generating units simultaneously. The $N+1$ principle ensures operation without power deficit with a probability of not less than $99.7 \%$ only with the most easy (i.e., ideal - unattainable) OCs for both GTUs (99.83\%) and GPUs (99.70\%). For the average $\mathrm{OC}$, the $N+1$ principle ensures an operation without power deficit only with a probability of not less than $95.4 \%$, that is, $98.57 \%$ for GTU and $95.99 \%$ for GPU. To obtain the probability of an operation without power deficit not less than $99.7 \%$ and medium OCs, the use of the $N+2$ principle is necessary. Similar conditions are for wind turbines with $98.65 \%$ and $99.95 \%$, respectively (regardless of the availability of wind as a primary energy source).

In order to achieve an acceptable balance reliability of power supply from the GPUs in the hardest OCs, it is necessary to use the $N+3(96.56 \%)$ principle to obtain a probability of an operation without power deficit not less than $95.4 \%$ and the $N+4(\sim 100 \%)$ principle for obtaining a probability of an operation without power deficit at least $99.7 \%$. It should be noted that the measures presented do not solve the issue of the reliability of the fuel transportation system. Essentially the conclusions about the necessary $N+n$ reserves to be based on the economic principle and backup costs analysis.

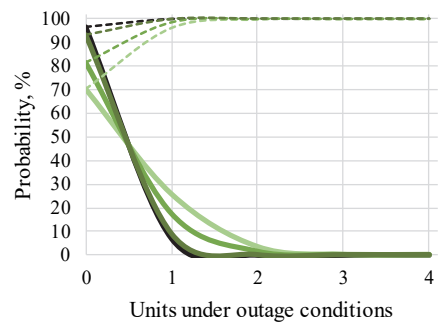

a)

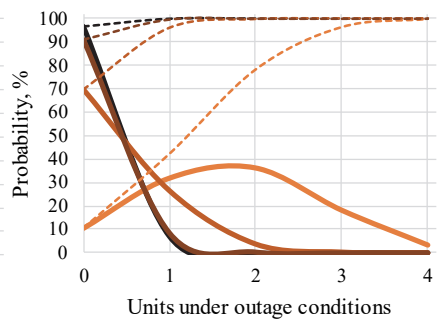

b)

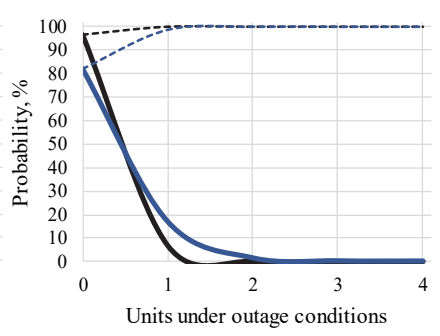

c)

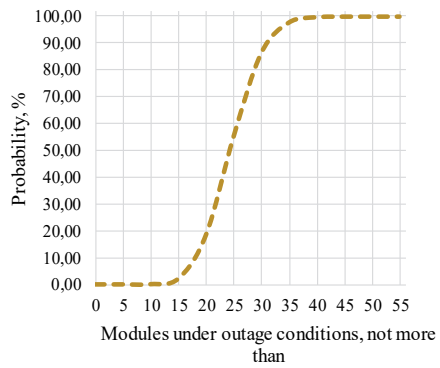

d)

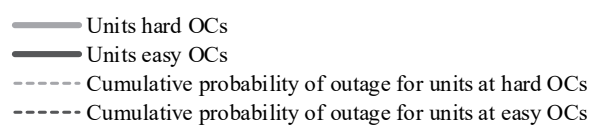

Fig. 2. Probability and cumulative probability of outage for: a) GTUs; b) GPUs; c) WPPs; d) PVPP modules 
For the photovoltaic station, the cumulative probability for the annual interval shows the need for an average of $1 \%$ of spare photovoltaic modules, which is 25 out of 2500 . Such a large number of reserve modules correlates with world statistics showing that the failure of one of the modules leads to a deterioration of conditions for the remaining modules of the entire string of the PVPP [16].

\section{Conclusions}

1. For hydrocarbon-based DG units, the increase in unit capacity within its range of effective capacities leads to an increase in reliability. For units based on renewables, an inversed relationship is observed, which is caused by the limiting capabilities of power semiconductor electronics.

2. Powerful modern wind turbines in general are inferior in reliability to units based on hydrocarbon fuel (even without regard to the availability of wind as a primary energy source). For photovoltaic plants, the novelty of the technical generation of photovoltaic modules plays a decisive role.

3. The DG units have $2-5 \%$ lower equipment availability factor in comparison with the units of large power plants. Nevertheless, DG units are subject to forced short-term outages 5-8 times often than the large generation units.

4. When calculating the reliability parameters of DG units, it is desirable to perform the statistics correction depending on the technical generation of units, the impact of internal and external causes of outages, and the expected electrical states.

5. The statistics described in the paper show that in the hardest operating conditions, the availability factor and the probability of operation deviate down from the mean ones by more than $40 \%$. Nevertheless, the mean time between forced outages during operation corresponds to the global one at 1300-2000 h. For most DG units in the world, it is due to the short electrical distance to the disturbance point in the grid and the excessive operation of imperfect relay protection devices.

6. The $N+1$ principle is limitedly applicable to the selection of the capacity and structure of the DG equipment. It provides a probability of an operation without power deficit of at least $99.7 \%$ (which is comparable to the grid's one) only for unattainable ideal operating conditions. Under medium and hard operation conditions depending upon the required probability of power deficit absence it is necessary to use up to $\mathrm{N}+4$ principle for the modern DG units. Essentially the conclusions about the necessary $N+n$ reserves to be based on the economic principle and cost analysis.

\section{Acknowledgement}

The work was supported by Act 211 Government of the Russian Federation, contract № 02.A03.21.0006.

\section{References}

[1] V.P. Oboskalov, Power Reserves in Power Systems. Sverdlovsk: Ural Polytechnikal Institution, 1989, 92 ps.

[2] V.P. Oboskalov, Technological aspects of power systems operation. Yekaterinburg, Ural Polytechnikal University, 2009, 194 ps.

[3] V.P. Oboskalov, Structural Reliability of Power Systems. Yekaterinburg, Ural Federal University, 2012, 196 ps.

[4] V.P. Oboskalov, An application of probabilistic methods and graph theory to electrical power engineering. Yekaterinburg, Ural Federal University, 2016, 271 ps.

[5] G. Celli, E. Ghiani, S. Mocci, and F. Pilo, "Distributed generation and intentional islanding: Effects on reliability in active grids", Proc. of the $18^{\text {th }}$ Int. Conf. and Exhibition on Electricity Distribution, CIRED 2005, Turin, Italy, 2005.

[6] C.V. Zeljkovic, N.L. Rajakovic, and S.J. Zubic, "Customerperspective approach to reliability evaluation of distributed generation", Proc. of the IEEE Trondheim PowerTech, Trondheim, Norway, 2011.

[7] M. Bahrami et al., "Predictive based reliability analysis of electrical hybrid distributed generation", Proc. of the Int. Conf. on Science and Technology (TICST), Pathum Thani, Thailand, 2015.

[8] J. Mitra, M.R. Vallem, and C. Singh, "Optimal Deployment of Distributed Generation Using a Reliability Criterion”, IEEE Trans. on Industry Applications, vol. 52, issue 3, pp. 1989 - 1997, MayJune 2016

[9] A. Heidari, V.G. Agelidis, H. Zayandehroodi, J. Pou, and J. Aghaei, "On Exploring Potential Reliability Gains Under Islanding Operation of Distributed Generation", IEEE Trans. on Smart Grid, vol. 7, issue 5, pp. 2166 - 2174, September 2016.

[10] A. Escalera, B. Hayes, and M. Prodanovic, "Analytical method to assess the impact of distributed generation and energy storage on reliability of supply", CIRED Open Access Proc. J., vol. 2017, issue 1, pp. 2092 - 2096, October 2017.

[11] P.V. Ilyushin, and Y.N. Kucherov "Approaches to estimation of consumers' reliable power supply using distributed generation objects", Electro. Electrical engineering, electric power industry, electrotechnical industry, vol. 5, pp. 2-7, 2014.

[12] Distributed Generation Operational Reliability and Availability Database. Energy and Environmental Analysis, Inc. Arlington, USA, 2004, 99 ps.

[13] S. Sheng, "Report on Wind Turbine Subsystem Reliability - A Survey of Various Databases", National Renewable Energy Laboratory, 2013, 43 ps.

[14] R.R. Hill, J.A. Stinebaugh, D. Briand, A.S. Benjamin, and J. Linsday, Wind Turbine Reliability: A Database and Analysis Approach, Albuquerque: Sandia National Laboratories, 2008, 72 ps.

[15] H.S. Huang, J.C. Jao, K.L. Yen, and C.T. Tsai, "Performance and Availability Analyses of PV Generation Systems in Taiwan”, Int. J. of Electrical, Computer, Energetic, Electronic and Communication Engineering, vol.5., 2011., pp. 731-735.

[16] A. Golnas, and S. Voss. PV System Reliability: An Operator's Perspective, SunEdison, 2013, 34 ps.

[17] T. Falin. Manual 19 Changes: Distributed Solar Generation in the Long-Term Load Forecast, PJM. 2015.

[18] A Kostyuchenko, "The Reliability Level of Gas Turbine Units", The Proc. of Public Periodic Sem. The Issues of DG Operation and Interconnection, Yekaterinburg, Russia, 2015.

[19] V.Y. Schaulov, "Experience of Development and Operation of CHP Based on Reciprocating Engines". The Proc. of the Small and Medium CHP Conf. Modern Solutions, Russia, Moscow, September 2005.

[20] N.D. Mukhlynin, A.V. Pazderin, A. Sukalo, "Estimating Operating Mode Parameters in Distribution Grids with Regard to the Peculiarities of Their Development", Proc. of the Int. Symp. on Industrial Electronics (INDEL 2016), Bosnia And Herzegovina, Banja Luka, November 2016. 